\title{
Sequences, geographic variations and molecular phylogeny of venom phospholipases and threefinger toxins of eastern India Bungarus fasciatus and kinetic analyses of its Pro31 phospholipases $A_{2}$
}

\author{
Inn-Ho Tsai ${ }^{1}$, Hsin-Yu Tsai ${ }^{1}$, Archita Saha ${ }^{2}$ and Antony Gomes ${ }^{2}$ \\ 1 Institute of Biological Chemistry, Academia Sinica, Taiwan, Institute of Biochemical Sciences, National Taiwan University, Taipei, Taiwan \\ 2 Department of Physiology, University of Calcutta, Kolkata, India
}

\author{
Keywords \\ Bungarus fasciatus; cDNA cloning; \\ phospholipase $\mathrm{A}_{2}$; phylogenetic analysis; \\ threefinger toxins; venom geographic \\ variation

\section{Correspondence \\ I.-H. Tsai, Institute of Biological Chemistry, Academia Sinica, Taiwan; Institute of Biochemical Sciences, National Taiwan University; POB 23-106, Taipei, Taiwan Fax: +886223635038

\begin{abstract}
Database
The sequence data were deposited in the GenBank database with the accession numbers: DQ508406, DQ508411-14 for KBf-VI, KBf-grlB, KBf-II, KBf-Va, and KBf-X, D0768745 for KBf-III, DQ835584 for Vb-2, respectively; DQ508407-10 for 3FTx-LI, -LK, -RK and -RI, and D0835582-3 for VIIla and 3FTX-LT, respectively
\end{abstract} \\ E-mail: bc201@gate.sinica.edu.tw
}

(Received 30 June 2006, revised 16 October 2006, accepted 17 November 2006)

doi:10.1111/j.1742-4658.2006.05598.x
Eight phospholipases $\mathrm{A}_{2}$ (PLAs) and four three-finger-toxins (3FTx) from the pooled venom of Bungarus fasciatus (Bf) were previously studied and sequenced, but their expression pattern in individual $\mathrm{Bf}$ venom and possible geographic variations remained to be investigated. We herein analyze the individual venom of two $\mathrm{Bf}$ specimens from Kolkata (designated as KBf) to address this question. Seven PLAs and five 3FTx were purified from the $\mathrm{KBf}$ venoms, and respective cDNAs were cloned from venom glands of one of the snakes. Comparison of their mass and $\mathrm{N}$-terminal sequence revealed that all the PLAs were conserved in both $\mathrm{KBf}$ venoms, but that two of their 3FTx isoforms were variable. When comparing the sequences of these KBf-PLAs with those published, only one was found to be identical to that of $\mathrm{Bf} \mathrm{Vb}-2$, and the other five were $94-98 \%$ identical to those of Bf II, III, Va, VI and XI-2, respectively. Notably, the most abundant PLA isoforms of $\mathrm{Bf}$ and $\mathrm{KBf}$ venoms contain Pro31 substitution. They were found to have abnormally low $k_{\text {cat }}$ values but high affinity for $\mathrm{Ca}^{2+}$. Phylogenetic analysis based on the sequences of venom group IA PLAs showed a close relationship between Bungarus and Australian and marine Elapidae. As the five deduced sequences of KBf-3FTx are only $62-82 \%$ identical to the corresponding Bf-3FTx from the pooled venom, the 3FTx apparently have higher degree of individual and geographic variations than the PLAs. None of the KBf-3FTx was found to be neurotoxic or very lethal; phylogenetic analyses of the 3FTx also revealed the unique evolution of $\mathrm{Bf}$ as compared with other kraits.
Snakes of the genus Bungarus are commonly known as kraits, which are characterized by their banded skin pattern. They are distributed from Pakistan through southern Asia to Indonesia and central China [1,2]. In the past, more than 20 proteins were purified and sequenced from pooled venom of Bungarus fasciatus
(Bf), which was obtained from either the Miami Serpentarium Laboratory or south-eastern Asia. The proteins include eight variants of phospholipases $\mathrm{A}_{2}$ (EC3.1.1.4, PLAs) [3-6], four isoforms of threefinger toxins (3FTx) [7-10], at least one Kunitz protease inhibitors [10-12], a factor-X activator [13], an

\section{Abbreviations}

$\mathrm{Bf}$, Bungarus fasciatus; diC ${ }_{16} \mathrm{PC}$, L-dipalmitoyl phosphatidylcholine; $\mathrm{diC}_{6} \mathrm{PC}$, L-dicaproyl phosphatidylcholine; $3 \mathrm{FTx}$, threefinger toxin; $\mathrm{KBf}$, Kolkata B. fasciatus; PLA, phospholipase $\mathrm{A}_{2}$. 
acetylcholine esterase [14] and other enzymes [15]. The numbers of isoforms for PLA and 3FTx from the pooled $\mathrm{Bf}$ venom were high, but the intraspecies or the geographic variations of this venom species have not been explored.

Intra-species variations of venom proteins [16] such as PLAs have been well documented for several viperid species [17,18], but are less well explored for elapid venom. In order to better understand the proteomics and variations of $\mathrm{Bf}$ venom, we studied individual venom of two specimens of Bf from Kolkata, India (designated as $\mathrm{KBf}$ ) by a comparative proteomic and genomic approach. The venom PLA and 3FTx isoforms were purified and characterized. After the mRNA was prepared from $\mathrm{KBf}$ venom glands, cDNAs corresponding to the two toxin families were amplified and cloned using specifically designed primers. The amino acid sequence and mass of the PLA and 3FTx were predicted from the cDNA sequences, matched with those of the purified $\mathrm{KBf}$ venom proteins as well as PLA and 3FTx isoforms reported for pooled Bf venom.

The three most abundant PLAs in Bf venom are $\mathrm{Va}, \mathrm{Vb}-2$ and VI (comprising $\sim 60 \%$ of the proteins in pooled venom); similar PLA isoforms are also abundant in the KBf venoms. These enzymes bear a Pro31 substitution near the highly conserved $\mathrm{Ca}^{2+}$ binding loop [19] and are characterized with low enzymatic activities [3], but show membrane-interfering activities and moderate lethality to mice [20,21]. By kinetic study, we further determined their abnormally low $k_{\text {cat }}$ values toward phospholipids substrates, but high $\mathrm{Ca}^{2+}$ binding affinity. Finally, phylogenetic analyses of the elapid PLAs and the krait 3FTx were carried out to better understand the intrageneric and intergeneric variations of kraits and their position in the Elapidae biosystematics.

\section{Results and Discussion}

\section{Purification and characterization of venom proteins}

To assure that the observed proteins sequence variations between the individual and pooled $\mathrm{Bf}$ venom could be attributed to geographic variations, venom samples were collected from two KBf near Kolkata in different seasons for this study. Crude venom was dissolved in buffer and fractionated by Superdex G75 gel filtration on a Pharmacia FPLC system (Fig. 1). Eluted fractions were collected and lyophilized separately. Pooled fractions $\mathrm{B}$ and $\mathrm{C}$ (Fig. 1) were then purified by reversed phase HPLC on a $\mathrm{C}_{18}$-column. The chromatographic profiles of the two $\mathrm{KBf}$ venoms were not identical (Fig. 2). Homogeneities of each protein peak were examined by SDS/PAGE. Abundance of a protein in the crude venom was estimated based on the relative peak area of its UV absorbance at $280 \mathrm{~nm}$ and expressed as percentage content $(\mathrm{w} / \mathrm{w})$, assuming equal extinction coefficient for all the proteins (Table 1).

A total of seven PLAs and five 3FTx were purified from each $\mathrm{KBf}$ venom, and were analyzed by automatic sequencing and mass spectrophotometry. The results were listed in Table 1. All these venom proteins showed a single mass peak by ESI-MS spectrometry, except that the PLA KBf-II contained a substantial amount of the oxidized form (13 019 Da)

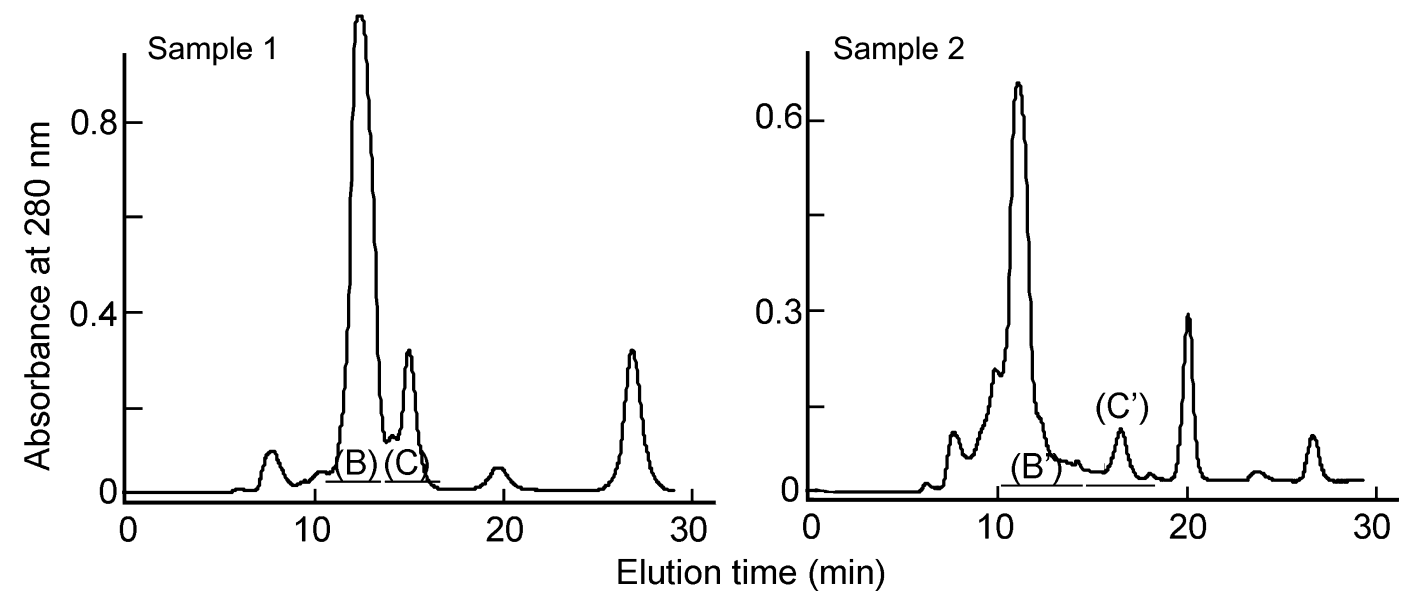

Fig. 1. Gel filtration of crude venoms of two KBf (samples 1 and 2). Venom powder (15-20 mg) of KBf was dissolved in $200 \mu \mathrm{L}$ of deionized water and loaded onto a Superdex G75 (HR10/30) column. The elution step was carried out on a FPLC system with an equilibration buffer containing $0.1 \mathrm{M}$ ammonium acetate $(\mathrm{pH} 6.24)$ at a flow rate of $0.5 \mathrm{~mL} \mathrm{~min}^{-1}$. Fractions (B), ( $\left.\mathrm{B}^{\prime}\right),(\mathrm{C})$ and $\left(\mathrm{C}^{\prime}\right)$ were pooled separately. 

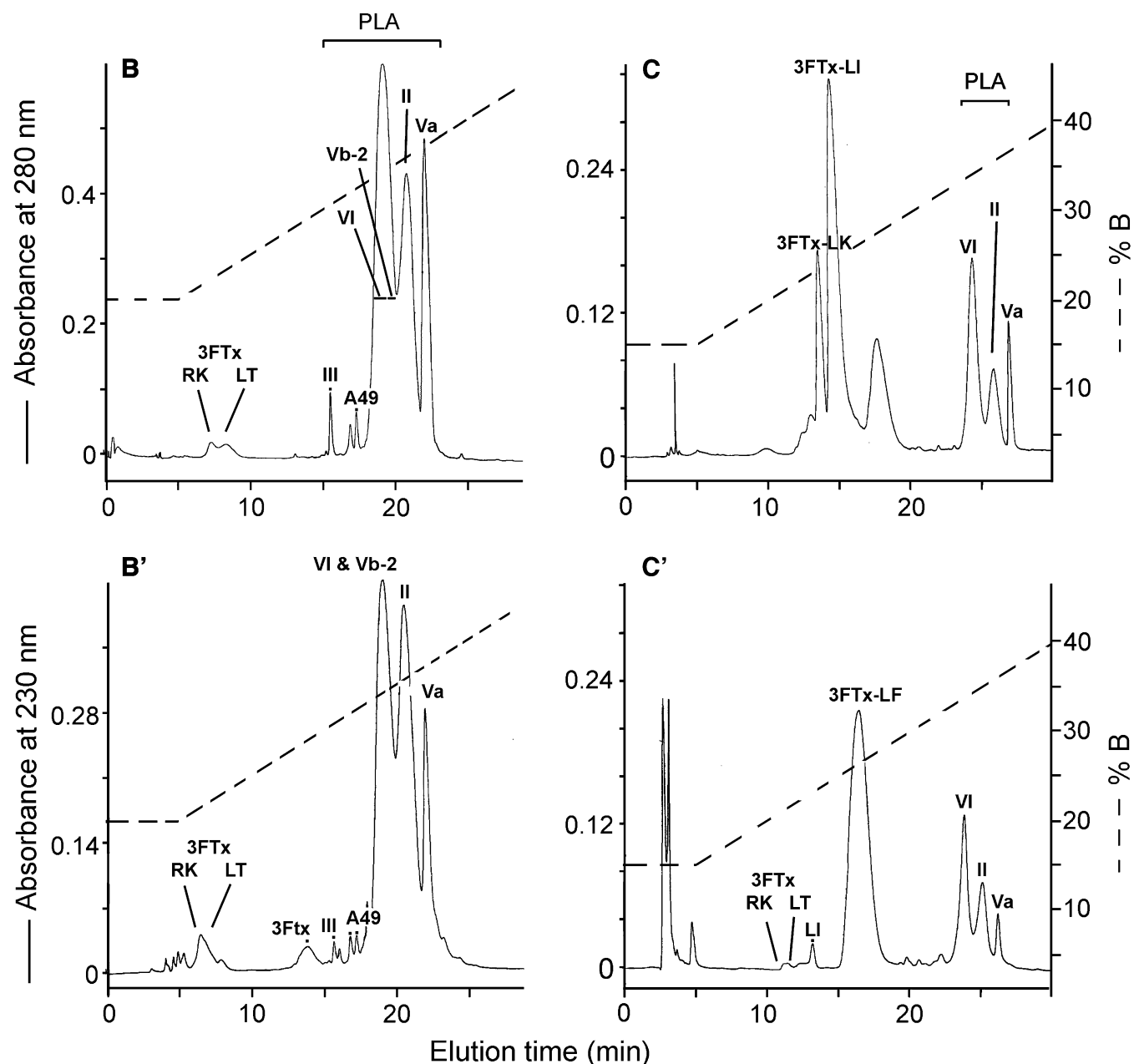

Fig. 2. Purification of venom proteins by RP-HPLC. Protein fractions from gel filtration were re-solubilized separately and injected into a Vydac RP-C18 column. For (B) and ( $\left.\mathrm{B}^{\prime}\right)$, elution started with $20 \%$ buffer B for 5 min followed by a linear gradient of buffer B for 25 min; for (C) and $\left(C^{\prime}\right)$, the elution started with $15 \%$ buffer $B$ for 5 min followed by a linear gradient of buffer B for $25 \mathrm{~min}$, flow rate was $1.0 \mathrm{~mL} \cdot \mathrm{min}{ }^{-1}$. Venom protein PLAs and 3FTx were purified and confirmed by ESI-MS and pH-stat enzyme assays. Their annotations are the same as in Table 1.

besides the native form (13003 Da). The KBf-PLAs were also matched with previously reported PLA variants of the pooled Bf venom [3-6]; only one of them was found to be identical to $\mathrm{Vb}-2$, with the others being $94 \%$ similar to the other five Bf PLA isoforms (Fig. 3). Two inactive PLA homologs, with N-terminal sequence either identical to Bf Ala49-PLA [5] or with a single substitution Val3ILe, were purified from both $\mathrm{KBf}$ venoms. The differences in their molecular masses (Table 1) and HPLC elution time (Fig. 2) may be attributed to this single mutation at position 3. The novel PLAs were thus named after their orthologous or closest Bf-PLA isoforms as: KBf-Va, KBf-VI, KBf-Vb-1, KBf-II, KBf-III, and KBf-A49, respectively (Table 1). Like the pooled venom, Vb-2, KBf-Va, and KBf-VI together com- prised about $55-60 \%$ of the individual venom mass. Notably, two Bf-PLAs, X-1 (13 025 Da) and XI-2 (13 $342 \mathrm{Da})[4,10]$, were absent in both $\mathrm{KBf}$ venom, although a highly similar PLA (designated as $\mathrm{KBf}-\mathrm{X}$ ) was cloned (see next session).

Various 3FTx subtypes were purified from the two $\mathrm{KBf}$ venoms and annotated as 3FTx-LI, -LK, LF, -LT, -RK and -RI, respectively, according to their first and second amino acid residues (Table 1). The individual KBf venoms have identical sets of PLAs and several conserved 3FTx (3FTx-LT and 3FTx-RK), but two of their 3FTx show sequence and mass variations (Table 1). In particular, the major 3FTx-LI (-LK) in sample $1 \mathrm{KBf}$ and $3 \mathrm{FTx}-\mathrm{LF}$ in sample $2 \mathrm{KBf}$ were very different. PLAs and 3FTx are common elapid venom families and are known to undergo accelerated 
Table 1. Inventory of PLAs and 3FTx purified from KBf venom. Masses were determined by ESI-MS spectrometry. PLA annotations follow those previously published or cloned (PL-II, accession number AF387594).

\begin{tabular}{|c|c|c|c|}
\hline PLA or 3FTx & $\begin{array}{l}\% \text { content } \\
(w / w)\end{array}$ & Mass (Da) & $\mathrm{N}$-Terminal sequence determined \\
\hline \multicolumn{4}{|l|}{ Both KBf } \\
\hline $\mathrm{KBf} \mathrm{Va}$ & 11 & $13079 \pm 1$ & NLLOFKNMIQ CAGSRLWVAY \\
\hline $\mathrm{Vb}-2$ & 15 & $13093 \pm 1$ & NLLQFKNMIQ CAGSRLWVAY \\
\hline $\mathrm{KBf} \mathrm{VI}$ & 23 & $13051 \pm 1$ & NLYQFKNMIE CAGTRTWLAY \\
\hline KBf II & 7 & $13003 \pm 1$ & NLLQFKNMIE CAGTRTWMAY \\
\hline KBf-III & 0.7 & $13412 \pm 1$ & NLFOFKNMIQ CAGTRSWTDY \\
\hline KBf-A49 & 0.6 & $13170 \pm 1$ & NMIOFKSMVO CTSTRPWLDY \\
\hline KBf-A49' & 0.4 & $13156 \pm 1$ & NMVQFKSMVOCTSTRPWLDY \\
\hline \multicolumn{4}{|c|}{ kBf, number 1} \\
\hline 3FTx-LI & 5.5 & $6455 \pm 1$ & LICYSSSMNKDSKT \\
\hline 3FTx-LK & 1.9 & $6401 \pm 1$ & LKCHTTOFRNIET \\
\hline 3FTx-LT\$ & 0.4 & $7421 \pm 1$ & LTCLICPEKYCOKVHTXR \\
\hline VIIlat & 0.4 & $7420 \pm 1$ & LTCLICPERYCOKVHTXR \\
\hline 3FTx-RK & 0.5 & $7305 \pm 1$ & RKCLTKYSODNESSKT \\
\hline \multicolumn{4}{|c|}{ kBf, number 2} \\
\hline 3FTx-LI & 0.1 & $6374 \pm 1$ & LICYSSPMSKETKTCQKWET \\
\hline $3 F T x-L F$ & 2.4 & $6882 \pm 1$ & LFCYKTPSTKGYQICEKWOT \\
\hline $3 F T x-L T^{a}$ & 0.5 & $7421 \pm 1$ & LTCLICPEKYCOKVHT \\
\hline VIIIa & 0.5 & $7420 \pm 1$ & LTCLICPERYCOKVHT \\
\hline 3FTx-RK & 1.2 & $7305 \pm 1$ & RKCLTKYSQDNESSKT \\
\hline
\end{tabular}

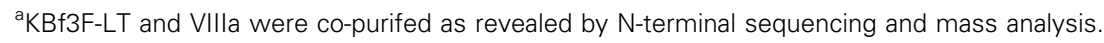

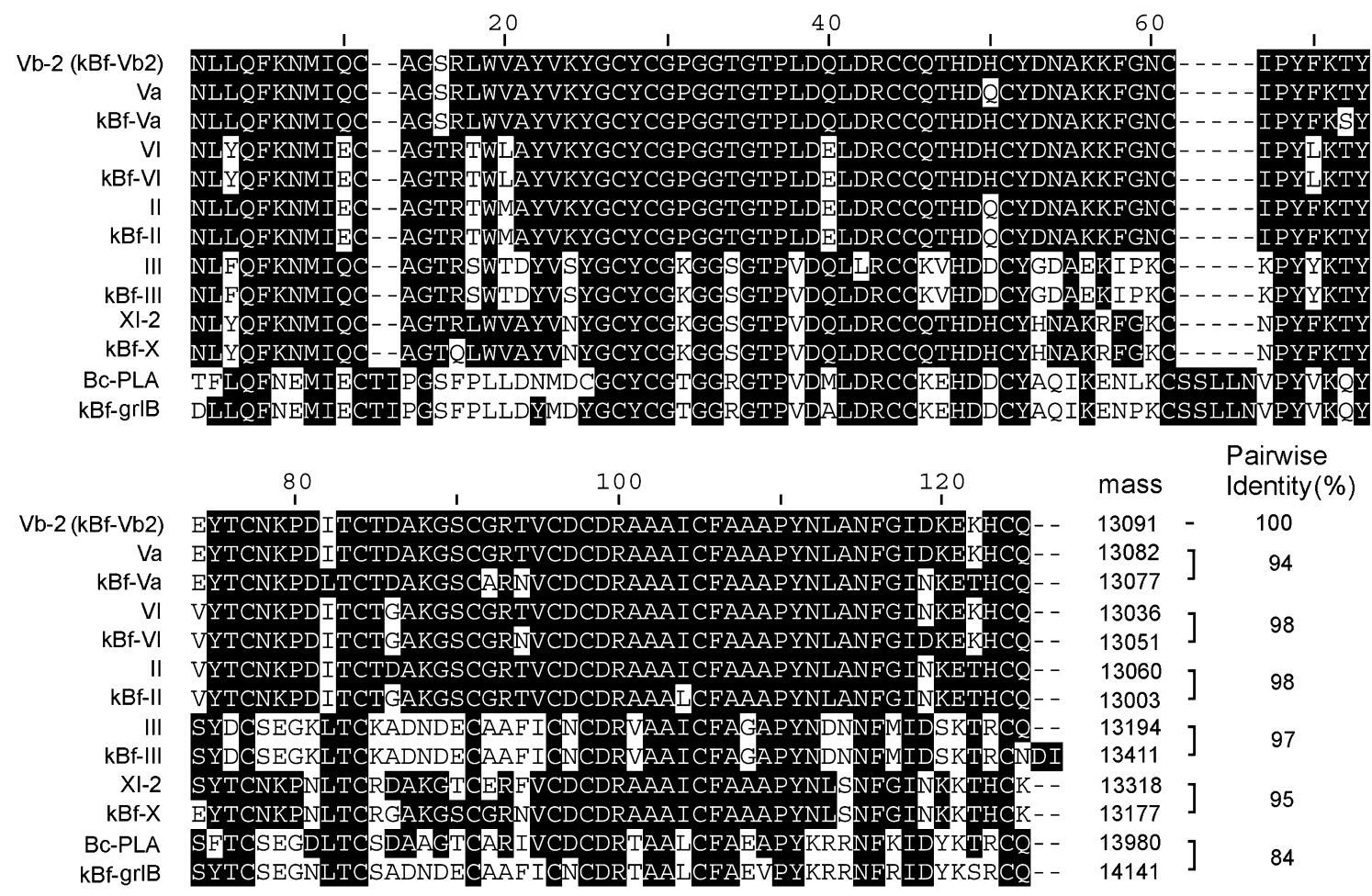

Fig. 3. Alignment of amino acid sequences of KBf PLAs and related venom PLAs. Single-letter codes of amino acids are used, conserved residues are reversed out, and gaps are marked with hyphens. The numbering system of Renetseder et al. [58] has been adopted. Accession numbers for B. fasciatus PLAs are as follows: Vb-2, P00609; Va, P00628; VI, P00627; II, O90WA8; III, P14615; for B. candidus group IB, GenBank AAO84769. 
evolution [22]. Intra-species venom variations usually result from quantitatively differential expression or minor structural changes of the venom proteins [18]. It is rather surprising that the venom 3FTx showed such a high degree of individual variation in the $\mathrm{KBf}$ specimens. Our results thus suggested that mutational rates of the exon of the 3FTx genes are much faster than those of the PLA genes, leading to high variation of KBf-3FTx.

\section{Cloning and cDNA sequencing}

Venom glands of only one of the $\mathrm{KBf}$ specimens were used for total RNA extraction. We have used facile methods to clone many toxin cDNAs from the $\mathrm{Bf}$ venom glands after cDNAs corresponding to the major toxin families had been amplified by PCR. This is a relatively economical and efficient approach to clone and determine protein sequences of the toxin families. It is also a powerful tool to study tissue-specific mRNAs expressed in low levels. Distinct clones were selected and sequenced at least twice, and then translated into amino acid sequences. Seven PLA clones were identified from about 50 sequenced cDNA clones and their full amino acid sequences were thus deduced (Fig. 3). The venom PLA precursors contain a conserved 27-residue signal peptide which is similar to those of other elapid venom PLAs (Table 2). The predicted enzyme regions also closely matched masses and partial sequences of the purified PLAs (Table 1). Using the same approach, a total of six 3FTx were cloned, sequenced and matched with the protein purified.
Their 21-residue signal peptides were also very conserved (Table 2).

Although two KBf-A49 as well as KBf-Vb-1 venoms were purified (Table 1), we failed to clone their cDNA. There are probably some distinct mutations in 5'-UTR of the cDNA templates, leading to insufficient priming during the PCR reactions. The Ala49 mutants are rather unique among the elapid venom PLAs, and mutations of Asp49Ala, Tyr28Asn and Gly30Asp at their catalytic $\mathrm{Ca}^{2+}$ binding sites [5] presumably abolish the enzymatic activity of KBf-A49 (Table 3). Nevertheless, we have cloned a group IB PLA (with pancreatic loop) and designated it as KBf-grIB. Its protein sequence is $84 \%$ identical to the group IB PLA cloned from the Malayan krait Bungarus candidus [23] (Fig. 3). The group IB PLAs were never been purified from Bungarus venoms, possibly because of degeneration.

Table 3. Enzymatic activities of purified venom PLAs toward zwitterionic micellar substrates. Initial hydrolysis rate of $3 \mathrm{mM} \mathrm{diC}_{16} \mathrm{PC}$ in the presence of $6 \mathrm{~mm}$ Triton $\mathrm{X}-100,10 \mathrm{mM} \mathrm{CaCl}_{2}$ and $0.1 \mathrm{M} \mathrm{NaCl}$ was measured with a pH-stat apparatus. Data of $\mathrm{Vb}-2$ and $\mathrm{VI}$ were taken from [3].

\begin{tabular}{lc}
\hline PLA & Specific activity $\left(\mu \mathrm{mol} \cdot \mathrm{mg}^{-1} \cdot \mathrm{min}^{-1}\right)$ \\
\hline KBf-Va & 23 \\
Bf-Vb-2 & 27 \\
Bf-VI & 9.8 \\
KBf-II & 25 \\
KBf-A49 & $<0.5$ \\
KBf-III & 45 \\
\hline
\end{tabular}

Table 2. cDNA deduced venom PLAs and $3 \mathrm{FTx}$ of $\mathrm{KBf}$. The isoelectric point (pl) and molecular mass were predicted from each protein sequence. ND, not determined.

\begin{tabular}{|c|c|c|c|c|}
\hline $\begin{array}{l}\text { Encoded } \\
\text { protein }\end{array}$ & $\begin{array}{l}\text { Calculated } \\
\text { mass (Da) }\end{array}$ & $\begin{array}{l}\text { Predicted } \\
\mathrm{pl}\end{array}$ & $\begin{array}{l}\text { Number } \\
\text { of clones }\end{array}$ & Signal peptide sequence \\
\hline \multicolumn{5}{|l|}{ PLA } \\
\hline $\mathrm{KBf}-\mathrm{Va}$ & 13077 & 8.0 & 4 & MYPAHLLVLLAVCVSLLGAANIPPQPL \\
\hline $\mathrm{Vb}-2$ & 13091 & 8.0 & 5 & MYPAHLLVLLAVCVSLLGAANIPPQPL \\
\hline KBf-VI & 13051 & 8.0 & 7 & MYPAHLLVLLAVCVSLLGAANIPPQSL \\
\hline KBf-II & 13003 & 8.0 & 2 & MYPAHLLVLLAVCVSLLGAANIPPOSL \\
\hline KBf-III & 13411 & 5.3 & 5 & ND \\
\hline $\mathrm{KBf}-\mathrm{X}^{\mathrm{a}}$ & 13177 & 8.9 & 2 & MYPAHLLVLLAVCVSLLGAANIPPQPL \\
\hline$K B f-g r l B^{a}$ & 14141 & 4.8 & 3 & MYPAHLLVLLAVCVSLLGAS I IPPOPL \\
\hline \multicolumn{5}{|r|}{ 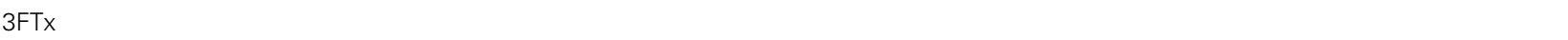 } \\
\hline 3FTx-LI & 6455 & 8.2 & 5 & MKTLLLTLVVVTIVCLDLGYT \\
\hline $3 F T x-L K$ & 6401 & 8.7 & 4 & MKTLLLTLVVVTIVCLDLGYT \\
\hline $3 F T x-L T$ & 7421 & 8.7 & 2 & MKTLLLTLVVVTIVCLDLGYT \\
\hline VIIla & 7420 & 8.7 & 9 & MKTLLLTLVVVTIVCLDLGYT \\
\hline $3 F T x-R K$ & 7305 & 9.5 & 1 & MKTLLLTLVVVTIVCLELGYT \\
\hline $3 F T x-\left.R\right|^{*}$ & 6968 & 8.7 & 3 & MKTLLLTLVVLTIVCLDLGHT \\
\hline
\end{tabular}

${ }^{\mathrm{a} C}$ Could not be isolated from both $\mathrm{KBf}$ venoms. 


\section{Alignment and comparison of amino acid sequences}

Complete amino acid sequences of KBf-PLA paralogs deduced from cDNA sequences were aligned pairwise with those of Bf-PLAs obtained by protein sequencing (Fig. 3). Apparently, only one of the KBf PLAs is identical to the previously reported $\mathrm{Vb}-2$, while the other five are $\sim 94-98 \%$ identical to Bf Va, Vb-1, VI, XI-2 (or X-1) [3,5,10] and PL-II (from Chinese Bf, accession number AF387594), respectively. Although its cDNA has been cloned, $\mathrm{KBf}-\mathrm{X}$ is not expressed in both $\mathrm{KBf}$ venoms. The previously reported $\mathrm{X}-1$ and $\mathrm{XI}-2$ [10] are structurally very similar to KBf-X and they possibly represent the allelic variants of $\mathrm{KBf}-\mathrm{X}$ in different individual snakes.

We also deduced the full protein sequences of five KBf-3FTx from cDNA sequences (Table 2). The KBf3FTx are all basic proteins with 57-62 amino acid residues and four disulfide bonds, except 3FTx-LT and VIIIa, which contain 65 residues and a fifth disulfide bond in the loop I region. The venom 3FTx of Bf and $\mathrm{KBf}$ may be putatively classified into five types with distinct N-terminal sequences (i.e. LI, LK, LT, RK or RI). They were aligned and compared with those of the 3FTx purified from the pooled Bf venom [7,8,10], or the most related sequences identified by a BLAST search (Fig. 4). Notably, only VIIIa is conserved in both $\mathrm{KBf}$ and $\mathrm{Bf}$ venom samples; the amino acid sequences of the other four KBf-3FTx appeared to be $62-82 \%$ identical to the published sequences of Bf-IV, fasiatoxin, VIIIa, and VII, respectively. Besides many amino acid substitutions, KBf 3FTx-LI and 3FTx-LK are shorter than their apparent Bf-3FTx orthologs (IV and fasciatoxin, respectively) by five or six residues at the C-terminus (Fig. 4). Thus, geographic variations of 3FTx are greater than those of PLAs in this venom species. Notably, all the four-disulfide-containing 3FTx of this species include a Trp residue at their loop II (Fig. 4), which is rather uncommon among elapid venom 3FTx [24]. We also found that 3FTx-LT is identical to a weak neurotoxin NTX4 (AY611643) present in B. candidus venom, while 3FTx-RK is $84 \%$ identical to bucain [25] of B. candidus.

\section{Calcium binding and kinetic parameter of the P31-PLAs}

Four PLAs (Va, Vb-2, VI and II) of KBf and Bf contain a Pro at position 31 and are hereafter referred to as P31-PLAs. Their functions appear to resemble cobra 'direct lytic factors' or cytotoxins, which cause membrane depolarization, muscle necrosis and moderate lethality $[20,21,26]$. These enzymes showed very low hydrolytic activities toward various kinds of micelles and mono-dispersed substrates in vitro (Table 3) [8,27]. Other P31-PLAs were also found in Australian and marine elapid venoms, including Pa-13, Pa-15 from Pseudechis australis, pseudexin B from Pseudechis porphyriacus [28-30], and LcPLH from Laticauda colubrina [31]. They are usually abundant in the venom and show low catalytic activities. Thus, the evolution of P31-PLAs in elapid venom bears a similarity to the Lys49-PLAs [32] in pitviper venom in the sense that they are all basic PLAs present in relatively high content and retain interfacial or membrane binding properties in spite of the low catalytic activities.

In fact, many of the inactive Lys49 PLAs from crotalid venoms also contain Pro31 [32], while other viperid venom PLAs usually contain Trp31 [17,32]. Group IA or elapid venom PLAs with higher catalytic activities usually contain Lys, Arg or Leu at position $31[33,34]$. Previous studies of pancreatic PLA mutants revealed that replacements of Leu31 or Arg31 by other amino acids reduced the enzymatic activities considerably [34,35]. Position 31 is at the entrance of the substrate cleft and is one of the major interface-recognition sites of PLAs [19,32,36]. It is thus reasonable to speculate that Pro31 substitution may affect either $\mathrm{Ca}^{2+}$ binding and/or configuration of the oxyanion-hole at the amide

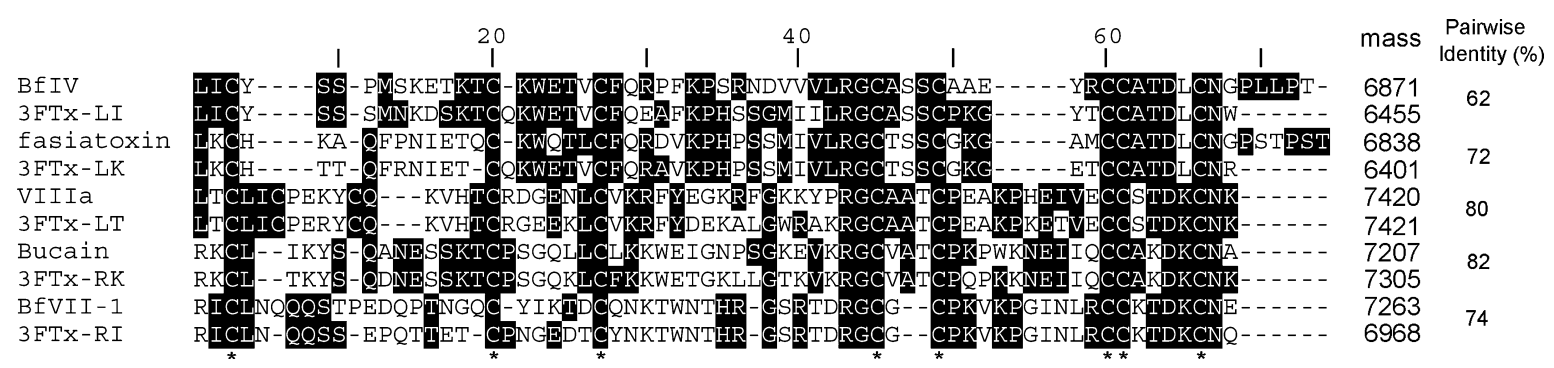

Fig. 4. Alignment of amino acid sequences of 3FTx of Bf and other related species. Single-letter codes of amino acids are used, conserved residues are reversed out, and gaps are marked with hyphens. Asterisks denote the eight conserved Cys residues. Swiss Prot accession numbers or references are as follows: fasicatoxin, P14534; VII-1, P10808; VI and VIIla [10], bucain (from B. candidus venom), P83346. 


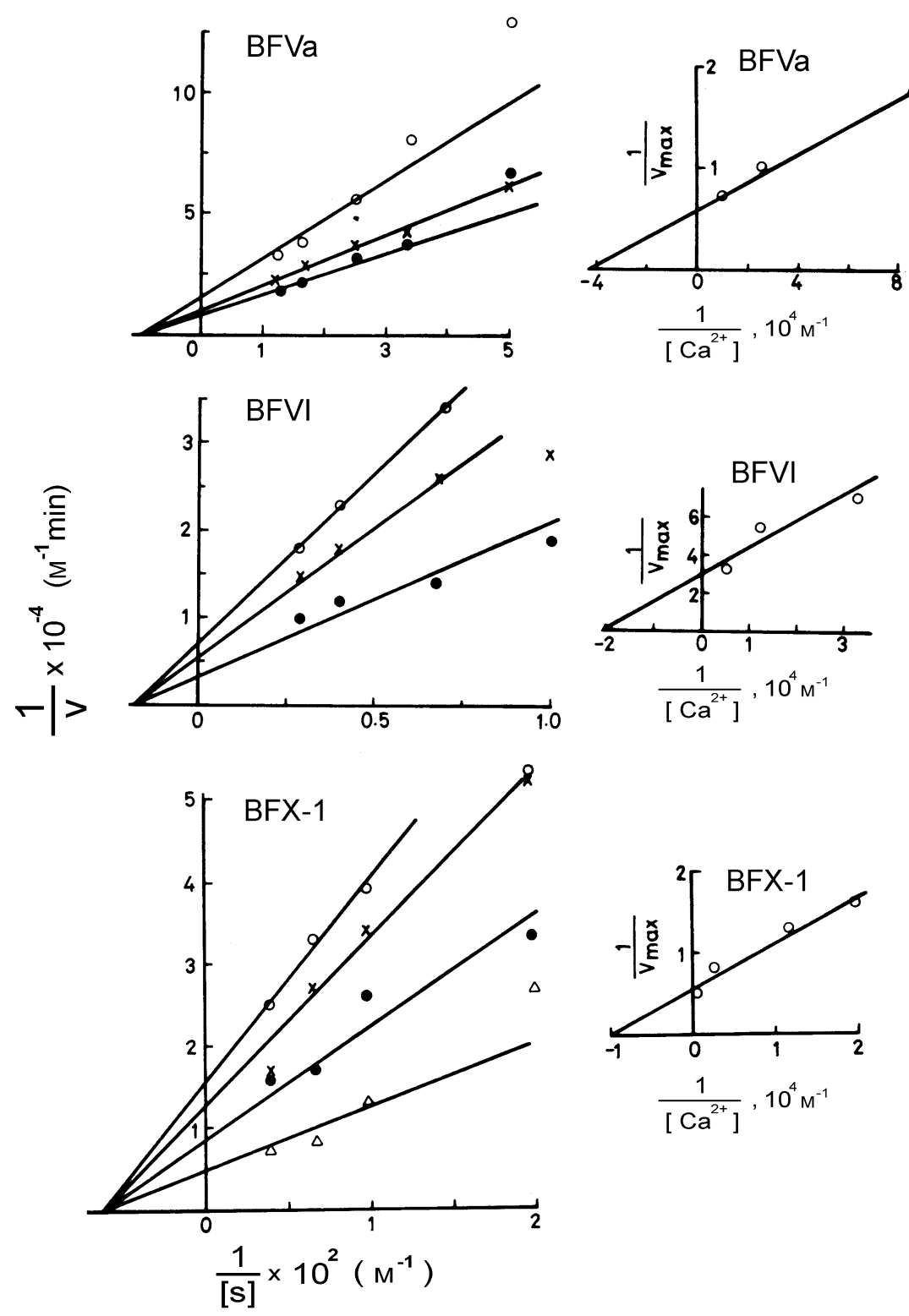

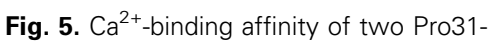
PLAs (Bf Va and VI) and a K31-PLA (Bf-X-1). The initial rate of hydrolysis of $3 \mathrm{~mm}$ $\mathrm{diC}_{16} \mathrm{PC}$ in the presence of $6 \mathrm{~mm}$ Triton $\mathrm{X}-100$ was measured by $\mathrm{pH}$-stat at $\mathrm{pH} 7.3$ and $37^{\circ} \mathrm{C}$ with $0.1 \mathrm{M} \mathrm{NaCl}$ at different $\mathrm{CaCl}_{2}$ concentrations. The $1 / V_{\max }$ values determined from double reciprocal plots were further plotted against reciprocals of $\mathrm{CaCl}_{2}$ concentrations to determine the $\mathrm{Ca}^{2+}$ affinity of the PLA.

backbone of Gly30 and thus the kinetic properties of the PLA reactions.

To better understand whether the $\mathrm{Ca}^{2+}$ binding was affected by Pro31 substitution, we carried out kinetic analyses of the P31-PLAs at different concentrations of $\mathrm{CaCl}_{2}$ (Fig. 5). Our results showed that the P31PLAs can bind $\mathrm{Ca}^{2+}$ with a dissociation constant of 13-49 $\mu \mathrm{M}$, suggesting a stronger binding than many other catalytically active venom PLAs, which have a $\mathrm{Ca}^{2+}$ dissociation constant of $100 \mu \mathrm{M}$ (Fig. 5). We also compared the kinetic properties of Bf VI (a P31-PLA) with those of Bf X-1 (containing K31) using L-dipalmitoyl phosphatidylcholine $\left(\mathrm{diC}_{16} \mathrm{PC}\right)$ in Triton $\mathrm{X}-100$ (1:2, molar ratio) and monodispersed L-dicaproyl phosphatidylcholine $\left(\mathrm{diC}_{6} \mathrm{PC}\right.$; Fig. 6B). The turnover rate $\left(k_{\mathrm{cat}}\right)$ of $\mathrm{Bf}$ VI calculated from double reciprocal plots was about 10-fold lower than that of $\mathrm{Bf} X-1$, while their apparent $K_{\mathrm{M}}$ values were rather similar (Fig. 6). Thus, it is very likely that the P31 substitution prevents the backbone amide of Gly30 from forming an essential oxyanion hole in the transition state, thus reducing $k_{\text {cat }}$ by $\sim 10$-fold.

The $\mathrm{Ca}^{2+}$-dependent hydrolysis of 2-acyl ester of lecithin substrate by P31-PLAs has been confirmed [37]. The enzymes have a preference to interact with the zwitterionic micelles $\left(\mathrm{diC}_{16} \mathrm{PC}\right.$ and Triton $\left.\mathrm{X}-100\right)$ rather than the anionic micelles $\left(\mathrm{diC}_{16} \mathrm{PC}\right.$ and deoxycholate) [3]. However, substrate binding to group I PLAs was 


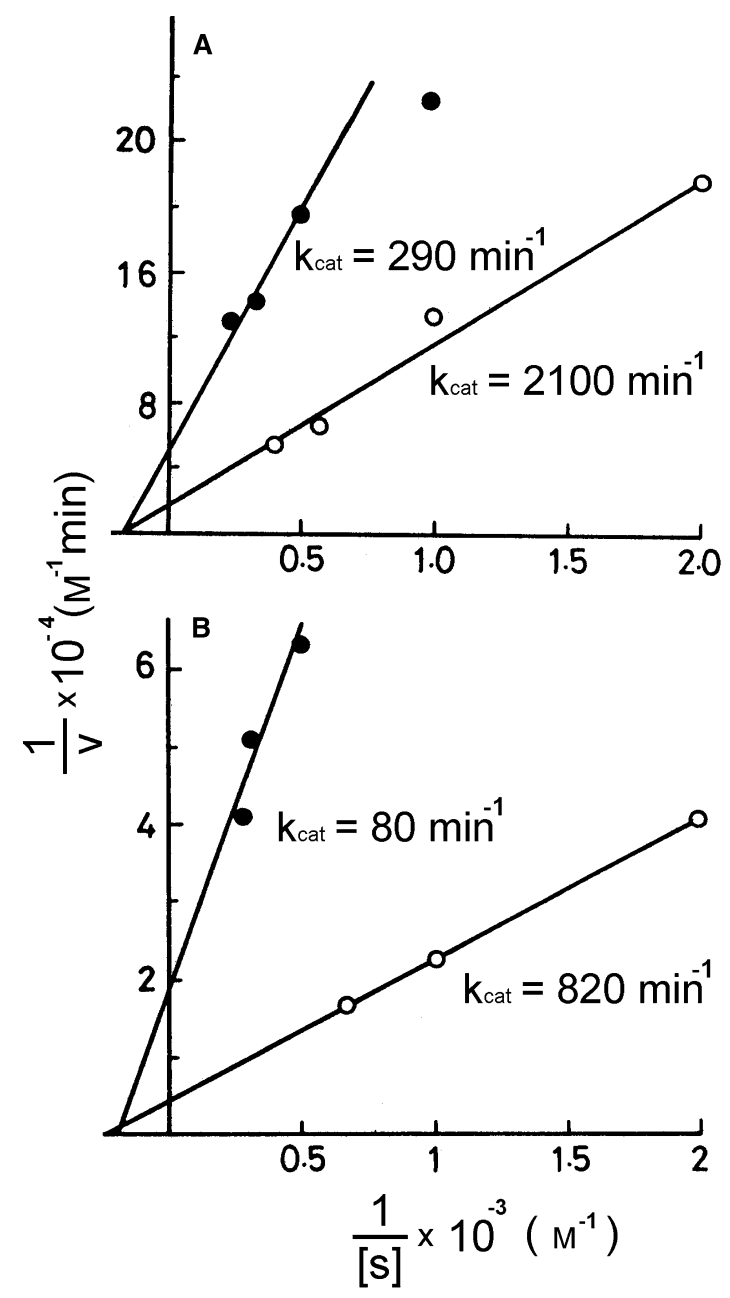

Fig. 6. Lineweaver-Burk plots of the hydrolysis of lecithins by Bf $\mathrm{VI}$ and $\mathrm{X}-1$. Initial reaction rates were measured by $\mathrm{pH}$-stat at $\mathrm{pH} 7.3$ and $37{ }^{\circ} \mathrm{C}$ with $0.1 \mathrm{M} \mathrm{NaCl}$ and $6 \mathrm{mM} \mathrm{CaCl} 2$. The value of $k_{\text {cat }}$ was calculated by dividing the $V_{\max }$ with the enzyme concentration. (A) Hydrolysis of mixed micelles of $\mathrm{diC}_{16} \mathrm{PC}$ and Triton $\mathrm{X}-100$ (1: 2); the PLA used was $0.14 \mu \mathrm{M} \mathrm{Bf} \mathrm{VI}(-)$ or $0.057 \mu \mathrm{M} B f \mathrm{X}-1$ (O). (B) Hydrolysis of $\mathrm{diC}_{6} \mathrm{PC}$; the PLA used was $1.4 \mu \mathrm{M} \mathrm{Bf} \mathrm{VI}($ or $0.57 \mu \mathrm{M} \mathrm{Bf} X-1(O)$, respectively.

found to be independent of the $\mathrm{Ca}^{2+}$ binding. This is in contrast with group II PLAs, whose substrate binding was facilitated $>10$-fold upon enzyme binding to $\mathrm{Ca}^{2+}$ [38]. It has been shown in other esterases that the contribution of the oxyanion hole to the transitionstate stabilization reaches $20 \mathrm{~kJ} \cdot \mathrm{mol}^{-1}$, and accounts for a 100 -fold increase of catalytic rates [39]. Because the P31-PLAs could effectively hydrolyze a chromogenic pseudo-substrate, 4-nitro-3-octanoyloxybenzoate [3], the transition state or mechanism of hydrolysis of this ester is probably different from that of the phospholipid micelles.

\section{Functions or toxicity of the $3 \mathrm{FTx}$}

The elapid venom 3FTx are a large multigene family and recent phylogenetic analyses of all the 3FTx revealed that kraits' venom may contain type I and II (short or long chain) $\alpha$-neurotoxins and many 'orphan groups' whose functional roles are not clear [40]. The major 3FTx in KBf (sample 1) are 3FTx-LI and -LK (i.e. 'orphan group XVIII'), which were either not at all or only weakly neurotoxic, as tested in pharmacological studies using the chick biventer cervicis [41] or rat phrenic nerve diaphragm [42]. Surprisingly, 3FTx-LI and -LK found in KBf sample 1 venom (Table 1) are not conserved in $\mathrm{KBf}$ sample 2 venom. The lethal dose $(\mathrm{LD})_{50}\left(2.1 \mathrm{mg} \cdot \mathrm{kg}^{-1}\right)$ for venom of number $1 \mathrm{KBf}$ used in this particular study was slightly higher than previously reported (1.3$1.5 \mathrm{mg} \cdot \mathrm{kg}^{-1}$ ) for the pooled venom from several suppliers [1]. Mice administered with a lethal dose of KBf venom did not show typical neurotoxic symptoms. The only postsynaptic neurotoxin previously isolated, albeit with low yield, from the pooled $\mathrm{Bf}$ venom was VII-1 [8] (belonging to type I $\alpha$-neurotoxin [40]), but we failed to isolate a similar protein from these two KBf venoms (Table 1). This can probably explain why the $\mathrm{KBf}$ venom has weaker lethality than the pooled $\mathrm{Bf}$ venom.

Notably, VIIIa and 3FTx-LT appears to be conserved in the venoms of both $\mathrm{KBf}$ and $\mathrm{Bf}$; they are similar to B. candidus NTX4 and Naja melanoleuca s4c11 (SwissProt P01400), which belong to the 'orphan group II' [40] or unconventional 3FTx [43]. Another protein $3 \mathrm{FTx}-\mathrm{RK}$ (belonging to 'orphan group III') is conserved in both $\mathrm{KBf}$ venoms, and is very similar to bucain from B. candidus venom [22] and a 3FTx cloned from Bungarus multicinctus (AJ006137 [44]). These 3FTx are present in moderate quantities and their targets remain to be identified. The fact that all isolated $\mathrm{Bf}$ venom proteins are less toxic $\left(\mathrm{LD}_{50}>4 \mu \mathrm{g} \cdot \mathrm{g}^{-1}\right.$ in mice) [10] than the crude venom $\left(\mathrm{LD}_{50}\right.$ of $1.3-2.1 \mu \mathrm{g} \cdot \mathrm{g}^{-1}$ in mice) suggests that synergisms between venom components are important.

\section{Phylogenetic analyses of krait PLAs and 3FTx}

The results in the present study suggested that previously reported Bf-PLA isoforms, including III, Va, Vb-2, VI, A49, and II (which was cloned from the Chinese $\mathrm{Bf}$ ), are probably paralogous to each other, as they coexist in a single $\mathrm{KBf}$ venom. A cladogram (Fig. 7) was built based on the amino acid sequences of 34 representative group IA PLAs with the king 


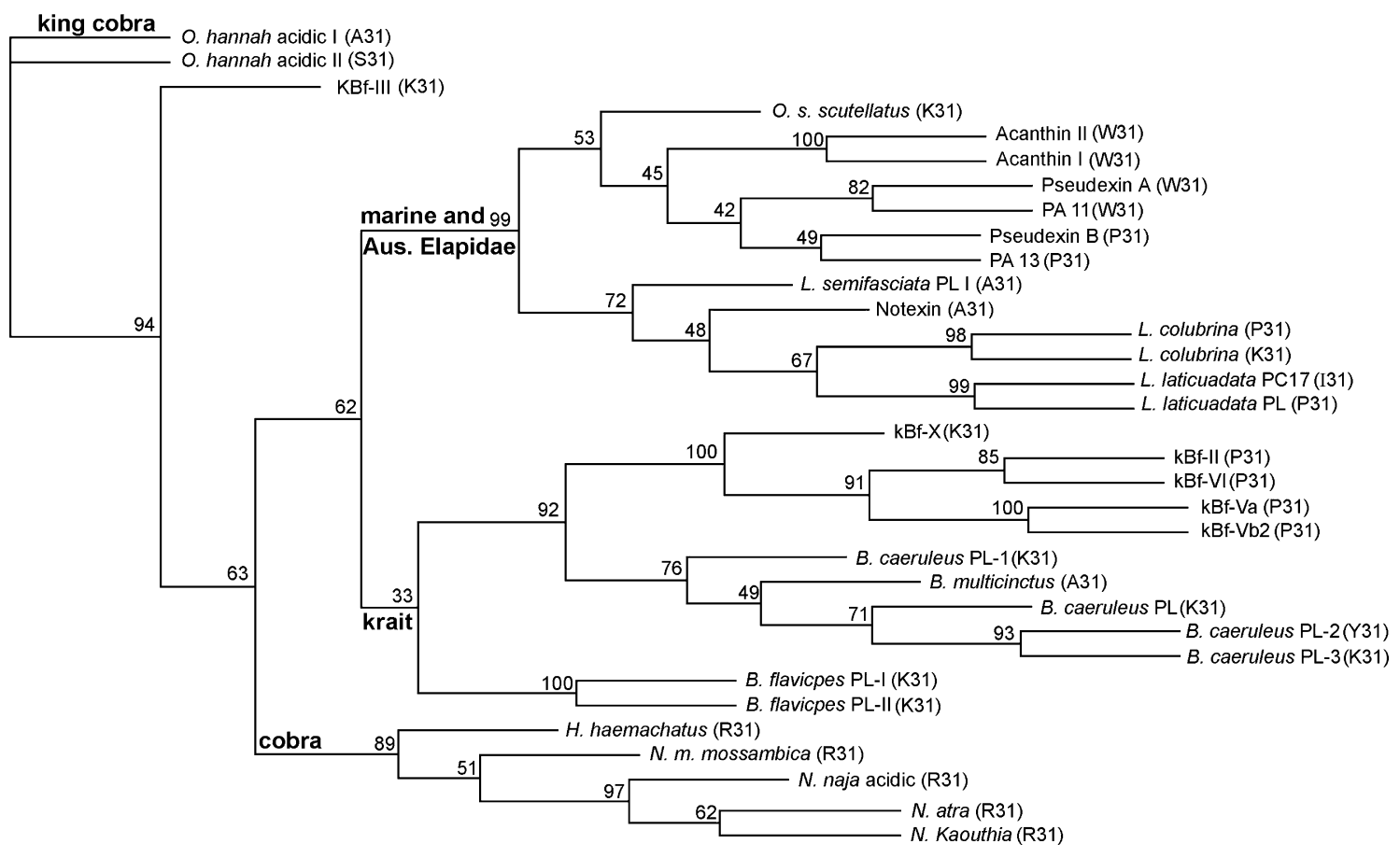

Fig. 7. Phylogenetic analysis of group IA venom PLAs. The dataset includes amino acid sequences of selected group IA elapid venom PLAs. Amino acid substitutions at position 31 were shown in parentheses. A group IB PLA purified from king cobra Ophiopagus hannah was used as the out-group. Values above the branches indicate the percentage of 1000 bootstrap replicates. Species names and accession numbers are as follows: Acanthophis antarcticus: acanthin I and II, P81236 and P81237; Bungarus caeruleus: PL, PL-1, -2 and -3, AF297663, AAS20530, AAR19228-9; Bungarus flavicpes: PL-I and -II, Ab112359-60; B. multicinctus: 0702209 A; Haemachatus haemachatus: P00595; Laticauda colubrina: K31 and P31, P10116 and P10117; Laticauda laticuadata: PC17 and PL, BAB72251 and CAA68449; Laticauda semifasciata: PL I, BAB72247; Naja atra: CAA51694; Naja kaouthia: P00596; Naja m. mossambica: P00602; Naja naja: acidic PLA, CAA45372; Notechis scutellatus: notexin, P00608; Oxyuranus scutellatus: OS2 AAB33760; P. australis: PA11 and PA13, P04056 and P04057; P. porphyriacus: pseudexin A and B, P20258 and P20259; and O. hannah: acidic I and II, P80966 and Q9DF33.

cobra venom group IB PLA as an out-group; all the KBf-PLAs except KBf A49 were included. The genus Bungarus appears to be monophyletic, as all the krait PLAs except KBf-III are allied together in this robust tree. Topology of this PLA tree is also in accord with a species tree based on the mtDNA sequences, showing that Bungarus contains three lineages represented by $\mathrm{Bf}$, Bungarus flaviceps and other Bungarus species, respectively $[1,40,45]$. Notably, venom PLAs of different genera of elapids are clearly resolved with high bootstrap supports in the phylogenetic tree (Fig. 7). The sea snakes have been shown $[45,46]$ to be diphyletic within the Australian and marine elapid clade (with the laticaudines and hydrophiines having separate origins). Notably, the PLA tree (Fig. 7) revealed that Bungarus is closer to Australian and marine elapid snakes than to the Asian cobra or king cobra; the relationship has not been shown in previous phylogenetic trees of elapid venom PLAs [45-47]. Our data thus support a novel phylogenetic relationship for reinterpretation of the systematics of these elapid genera.
In addition, a cladogram of kraits' 3FTx was built based on the amino acid sequences (Fig. 8). It has been pointed out that type I and type II $\alpha$-neurotoxins are ubiquitous among elapid venoms, but that orphan groups III, IV, V, IX, XVII, XVIII and XIX of 3FTx are restricted to kraits' venom [40]. The tree in Fig. 8 shows that $\mathrm{Bf}$ venom contains only four paralogous 3FTx, i.e. type I $\alpha$-neurotoxin and orphan groups II, III, and XVIII. In contrast, venoms of B. multicinctus, B. candidus and B. flaviceps have special type II $\alpha$ - and $\kappa$-neurotoxins [40,48] and orphan groups IV, V, IX, XVII, or XIX, while sharing the orphan groups II and III with Bf venom. Notably, the neurotoxic PLAs ( $\beta$-bungarotoxins) are present in venom of all kraits except $\mathrm{Bf}[48,49]$. It is thus likely that $\mathrm{Bf}$ is an unique and primitive krait lineage. Speciation of Bf possibly took place before the other kraits evolved distinct 3FTx-orphan groups and strong type II neurotoxins and $\beta$-bungarotoxins, and before B. flaviceps lineage split from other neurotoxic kraits including B. caelurus, B. multicinctus and B. candidus $[1,48]$. 


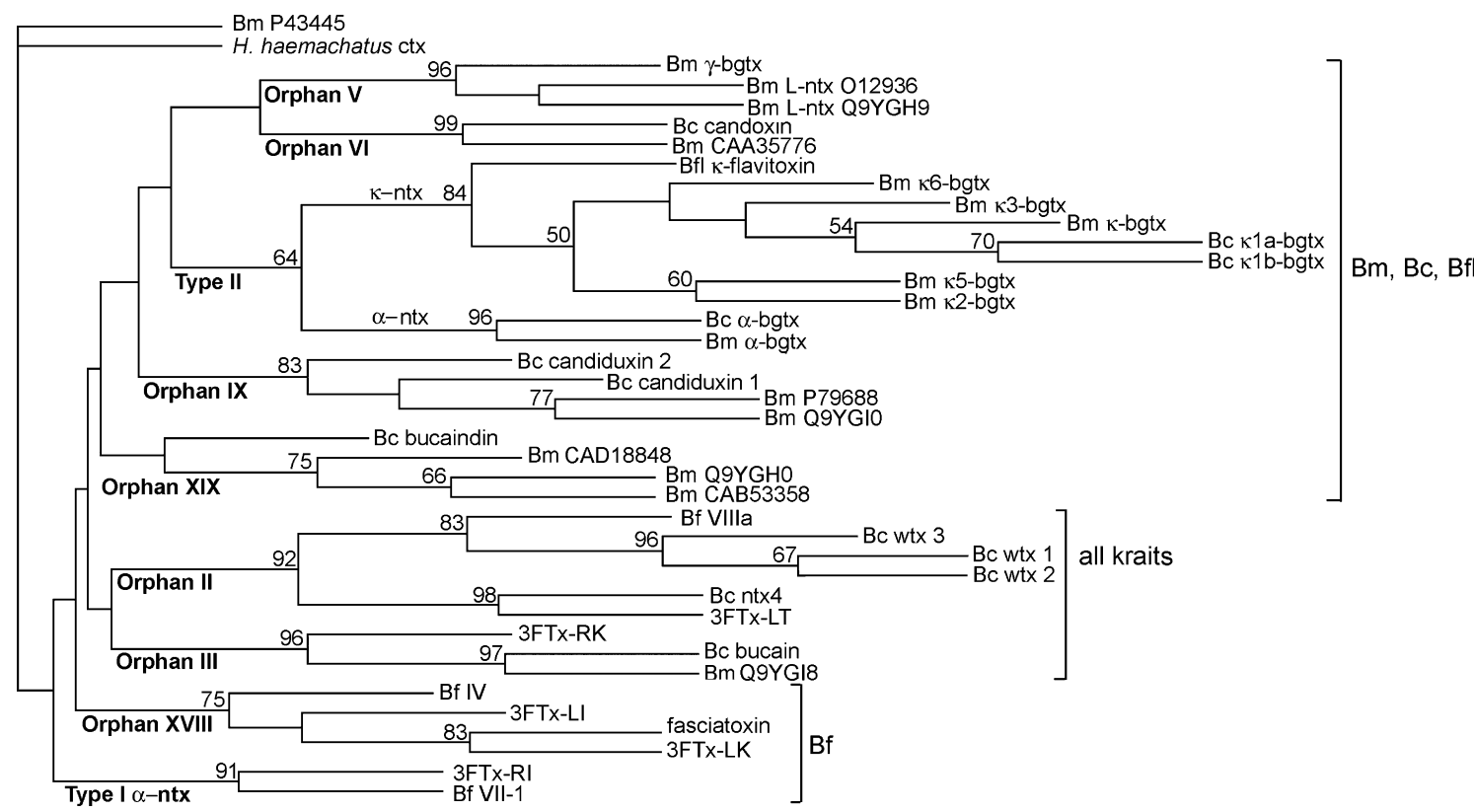

Fig. 8. Phylogenetic analysis of kraits' venom 3FTx. The dataset used includes full amino acid sequences so far available for $3 F T x$ of krait venoms, except a possibly erroneous Q9W727 [40]. H. haemachatus cytotoxin P24776 was used as the out-group. Values above branches indicate the percentage of 1000 bootstrap replicates. In addition to those directly shown in the tree, the accession numbers and references are as follows: B. candidus (Bc): $\alpha$-bgtx CAD92407, bucain P83346, bucaindin P81782, candiduxin 1 and 2 AAL30057 and 8, candoxin AAN16112, ntx4 AAT38875, wtx 1-3 AAL30059-61; B. flaviceps (Bfl): $\kappa$-flavitoxin P15815; B. multicinctus (Bm): $\alpha$-bgtx CAB51843, $\gamma$-bgtx CAD01082, к, к1a, к1b, к2, к3, к5, к6-bgtx CAA69971, AAL30054-5, P15816, CAA72434, 012962, Q9W729; and B. fasciatus (Bf): Bf-IV [59], BfVII-1 P10808, VIIla [10], fasciatoxin P14534. ntx, neurotoxin.

\section{Summary and conclusions}

Intrageneric and intraspecies variations of kraits' venom have been investigated by proteomic and transcriptomic analyses herein and in other recent studies $[40,48,49]$. We have cloned and sequenced from a KBf specimen a total of seven PLAs and six 3FTx KBf; among them 11 were novel sequences (Table 2). Major findings or conclusions from this study are: (a) Individual $\mathrm{Bf}$ venom contains almost as many paralogous PLAs and 3FTx variants as the pooled venom. (b) The small and nonenzymatic 3FTx show much greater geographic and individual variations than the PLAs in this venom species. (c) Pro31 substitution in "cardiotoxinlike PLAs' is an evolutionary strategy to reduce the enzyme turnover rates but retain high affinity for binding to $\mathrm{Ca}^{2+}$ and the membrane interface. (d) Kraits are possibly genetically related to Australian and marine elapids. (e) Bf venom has evolved distinct PLA and 3FTx subtypes which are not found in other kraits' venoms, and their functions remain to be elucidated. Apparently, Bf split from other krait species in very ancient times and evolved with non-neurotoxic venom strategy. It is also worth noting that the prey of $\mathrm{Bf}$ and king cobra consists mainly of snakes and reptiles, which are distinct from those of other kraits (e.g. B. candidus and B. multicinctus, which prey on small catfishes, eels and rodents [50]).

\section{Experimental procedures}

\section{Materials}

Crude venom was milked from two individual specimen of Bf (Calcutta Snake Park, Kolkata, India). Venom glands were dissected after killing one of the snakes. The tissue was preserved for several weeks in the RNAlater solution (Ambion, Austin, TX, USA) before extraction of mRNA for preparation of the cDNA. Modification and restriction enzymes and the pGEM-T vector were purchased from Promega (Madison, WI, USA). Phospholipid substrate was from Avanti-Biochemical (Alabaster, AL, USA). Triton X-100 and sodium deoxycholate were from Sigma Chemical Co. (St Louis, MO, USA). All buffers and chemicals were reagent grade.

\section{Venom protein purification}

Lyophilized venom $(15-20 \mathrm{mg})$ was dissolved in a small volume of $100 \mathrm{~mm}$ ammonium acetate (pH 6.24) followed 
by centrifugation at $9000 \mathrm{~g}$ for $5 \mathrm{~min}$ on a Kubota (Tokyo, Japan) KM-15200 centrifuge equipped with angle rotor RA2724. The supernatant was applied to a Superdex-G75 gel filtration column and eluted with the same buffer on a FPLC system. Fractions containing PLAs and 3FTx were further purified by reverse-phase HPLC on a Vydac C18 column (Vydac; $4.6 \times 250 \mathrm{~mm}$ ). Elution was carried out in a gradient containing buffers $\mathrm{A}$ and $\mathrm{B}$, which were made of $0.07 \%(\mathrm{v} / \mathrm{v})$ trifluoroacetic acid in distilled water and acetonitrile, respectively. Proteins collected from the elution peaks were dried in a vacuum-centrifuge device (Labconco, Kansas City, MO, USA). Protein concentrations in stock solutions were determined with a dye-based protein determination kit from Bio-Rad (Hercules, CA, USA) [51].

\section{Determination of protein sequences and masses}

The N-terminal sequences of purified proteins were determined by a gas-phase amino acid sequencer coupled with a phenylthiohydantoin amino acid analyzer (model 477 A; Perkin Elmer, Foster City, CA, USA). The molecular weight of each purified protein (dissolved in $0.1 \%$ acetic acid with $50 \%$ acetonitrile by volume) was analyzed under positive mode by ESI-MS on a mass spectrometer (Sciex API100, Perkin Elmer). Purity of the venom protein was assessed by SDS/PAGE and N-terminal sequencing.

\section{Cloning of venom toxins}

The mRNA from Bf venom glands was extracted using the mRNA extraction kit. Their complementary DNA (cDNA) was prepared using the cDNA synthesis kit according to the manufacturer's instructions (Stratagene, La Jolla, CA, USA). PCR primers were synthesized based on the conserved regions of the cDNA sequences encoding homologs of elapid venom PLAs [23] and 3FTx [24], respectively. For amplification of 3FTx, primer 1 was 5 -ATGAAAAC TCTGCTGCTGACCTTG-3' and primer 2 was 5'-CTCAA GGAAWTTAGSCAC TCRKAGAG-3'. For amplification of PLAs, the primers 3 and 4 used were $5^{\prime}$-GCAGTTTGT GTCTCCCTCTTAGGA-3' and 5'-CACAGTCCTTGA GCTGAAGCTTCTC-3'. In addition, primer 5 (5'CAG(C,T)(C,A)TCTCAATCTCTT(T,C)-3') was designed based on the N-terminal sequence of KBf-III to replace primer 3 for PCR. Primers 1, 3 and 5 were in the sense orientation of the $5^{\prime}$-end sequence, whereas primers 2 and 4 were in the antisense direction of a conserved region at the 3 '-end untranslated region.

PCR was conducted using cDNA of Bf venom glands as templates in the presence of SuperTaq DNA polymerase (HT Biotech, Cambridge, UK) [52]. The conditions of each of the 30 cycles were set to $92{ }^{\circ} \mathrm{C}$ for 1.0 min during denaturation, $52{ }^{\circ} \mathrm{C}$ for $1.0 \mathrm{~min}$ during annealing, and $72{ }^{\circ} \mathrm{C}$ for $1.0 \mathrm{~min}$ during extension. As examined by $1 \%$ agarose gel electrophoresis, DNA fragments at the expected size for PLA, 3FTx and $\mathrm{KuI}$ were specifically amplified. After treating with polynucleotide kinase, the product was inserted into the pGEM$\mathrm{T}$ vector (Promega Biotech) that was then used to transform Escherichia coli strain JM109 [53]. The plasmid DNA was extracted from white transformants and was further examined for its restriction pattern by agarose gel electrophoresis. The cloned cDNA was sequenced by the DNA-SequencingSystem (model 373 A; PE-Applied Biosystems, Foster City, CA, USA).

\section{PLA assay and kinetic analysis}

Micelles of $3 \mathrm{~mm} \mathrm{diC}{ }_{16} \mathrm{PC}$ with $3 \mathrm{~mm}$ sodium deoxycholate or $6 \mathrm{~mm}$ Triton $\mathrm{X}-100$, or $\mathrm{diC}_{6} \mathrm{PC}$ and $100 \mathrm{~mm} \mathrm{NaCl}$ were prepared in a glass-Teflon tissue homogenizer, and $2.5 \mathrm{~mL}$ of the solution was transfer to a reaction cup with a thermostat of the $\mathrm{pH}$-stat apparatus (Radiometer, Copenhagen, Denmark). With constant stirring, $10 \mathrm{~mm} \mathrm{CaCl}_{2}$ was added directly before addition of the enzyme. Release of acid during substrate hydrolysis was followed by $\mathrm{pH}$-stat titration at $\mathrm{pH} 7.4$ and $37^{\circ} \mathrm{C}$ with $8 \mathrm{~mm} \mathrm{NaOH}$. The initial hydrolysis rate was corrected to the nonenzymatic rate in each experiment. The affinity of $\mathrm{Ca}^{2+}$ was determined kinetically at different concentrations of $\mathrm{CaCl}_{2}$ following the published methods [3,38].

\section{Neuromuscular effects}

Neurotoxicity of purified $\mathrm{Bf}$-venom proteins was assessed on chick biventer cervicis [42] and rat phrenic nerve diaphragm neuromuscular preparation [43]. Leghorn chicks (10 days old) were anaesthetized with chloroform and the biventer cervicis muscle was dissected out. One end of the muscle was tied with a oxygenator tube and the other end was tied with Brodie's lever. The muscle was passed through the platinum electrode. The preparation was suspended in $4 \mathrm{~mL}$ oxygenated $\left(95 \% \mathrm{O}_{2}+5 \% \mathrm{CO}_{2}\right)$ Tyrode's solution $\left(137 \mathrm{~mm} \mathrm{NaCl}, 2.7 \mathrm{~mm} \mathrm{KCl}, 1.8 \mathrm{mM} \mathrm{CaCl}_{2}\right.$, $1.2 \mathrm{~mm} \mathrm{MgCl}, 11.9 \mathrm{~mm} \mathrm{NaHCO} 3,0.4 \mathrm{~mm} \mathrm{NaH} \mathrm{NO}_{4}$ and $5.5 \mathrm{~mm}$ glucose) at room temperature $\left(29 \pm 1{ }^{\circ} \mathrm{C}\right)$.

Male albino rats $(150 \pm 10 \mathrm{~g})$ were killed by stunning and the hemidiaphragm with attached phrenic nerve was dissected out with a small portion of the anterior chest wall to serve as an anchor for the platinum electrode. The pointed end of the diaphragm segment was attached to Brodie's lever with a thread and the nerve was threaded through the platinum electrode. The chick or rat preparation was suspended in $6 \mathrm{~mL}$ oxygenated Tyrode solution at room temperature $\left(29 \pm 1{ }^{\circ} \mathrm{C}\right)$. The preparation was stimulated with a square wave electronic stimulator at $8-12 \mathrm{~V}$ of $0.5 \mathrm{~ms}$ duration and 10 -s pulse. Muscle contractions were recorded by Brodie's lever on a rotating smoked drum. 


\section{Lethal effects}

Lethal potency of purified PLA was determined in ICR adult mice of $\sim 30 \mathrm{~g}$ body weight. The PLA was injected intraperitoneally with $0.1 \mathrm{~mL}$ protein prepared in sterile phosphate-buffered saline. Six mice were used to obtain the median $\mathrm{LD}_{50}$ of each dosage. $\mathrm{LD}_{50}$ and its confidence limit at $95 \%$ probability were calculated [54].

\section{Phylogenetic analysis of Bungarus venom $\mathrm{PLA}_{2}$}

The alignment of amino acid sequences was prepared using the CLLUSTAL w program [55]. Cladograms were constructed based on the aligned sequences by a neighbor-joining algorithm using the PHYLIP program [56], and the degree of confidence for the internal linage was determined by bootstrap methods [57].

\section{Animals}

Animals (mice, rats and chicks) were treated according to institutional guidelines for the care and use of experimental animals under the approval of the University of Calcutta, India.

\section{Acknowledgements}

This work was supported by grants from Academia Sinica and National Science Council, Taiwan, ROC. The authors thank C. S. Liu for his encouragement and advice, and for his generosity in providing B. fasciatus venom PLAs for kinetic studies.

\section{References}

1 Slowinski JB (1994) A phylogenetic analysis of Bungarus (Elapidae) based on morphological characters. $J$ Herpetol 28, 440-4462.

2 Tan NH \& Ponnudurai G (1990) A comparative study of the biological properties of krait (genus Bungarus) venoms. Comp Biochem Physiol 95C, 105-109.

3 Liu CS, Chen JM, Chang CH, Chen SW, Tsai IH, Lu HS \& Lo TB (1990) Revised amino acid sequences of the three major phospholipases $\mathrm{A}_{2}$ from Bungarus fasciatus (banded krait) venom. Toxicon 28, 1457-1468.

4 Liu CS, Leu HL, Chang CS, Chen SW \& Lo TB (1989) Amino acid sequence of a neutral phospholipase $\mathrm{A}_{2}$ (III) in the venom of Bungarus fasciatus. Int J Pept Protein Res 34, 257-261.

5 Liu CS, Kuo PY, Chen JM, Chen SW, Chang CH, Tseng CC, Tzeng MC \& Lo TB (1992) Primary structure of an inactive mutant of phospholipase $\mathrm{A}_{2}$ in the venom of Bungarus fasciatus (banded krait). J Biochem (Tokyo) 112, 707-713.
6 Liu CS, Chang CS, Leu HL, Chen SW \& Lo TB (1988) The complete amino-acid sequence of a basic phospholipase $\mathrm{A}_{2}$ in the venom of Bungarus fasciatus. Biol Chem Hoppe Seyler 369, 1227-1233.

7 Liu CS, Hsiao PW, Chang CS, Tzeng MC \& Lo TB (1989) Unusual amino acid sequence of fasciatoxin, a weak reversibly acting neurotoxin in the venom of the banded krait, Bungarus fasciatus. Biochem J 259, 153-158.

8 Liu CS, Chen JP, Chang CS \& Lo TB (1989) Amino acid sequence of a short chain neurotoxin from the venom of banded krait (Bungarus fasciatus). J Biochem (Tokyo) 105, 93-97.

9 Jiang MS, Haggblad J \& Heilbronn E (1986) Interaction with chick myotube cholinergic receptors of an alphaneurotoxin isolated from venom of the banded krait (Bungarus fasciatus). Toxicon 24, 713-719.

10 Liu CS \& Lo TB (1994) Chemical studies of Bungarus fasciatus venom. J Chinese Biochem Soc 23, 69-75.

11 Liu CS, Wu TC \& Lo TB (1983) Complete amino acid sequences of two protease inhibitors in the venom of Bungarus fasciatus. Int J Pept Protein Res 21, 209 215.

12 Chen C, Hsu CH, Su NY, Lin YC, Chiou SH \& Wu SH (2001) Solution structure of a Kunitz-type chymotrypsin inhibitor isolated from the elapid snake Bungarus fasciatus. J Biol Chem 276, 45079-45087.

13 Zhang Y, Xiong YL \& Bon C (1995) An activator of blood coagulation factor $\mathrm{X}$ from the venom of Bungarus fasciatus. Toxicon 33, 1277-1288.

14 Cousin X, Creminon C, Grassi J, Meflah K, Cornu G, Saliou B, Bon S, Massoulie J, Bon C, Cousin X et al. (1996) Acetylcholinesterase from Bungarus venom: a monomeric species. FEBS Lett 387, 196-200.

15 Yost DA \& Anderson BM (1983) Adenosine diphosphoribose transfer reactions catalyzed by Bungarus fasciatus venom NAD glycohydrolase. J Biol Chem 258, 3075-3080.

16 Warrell DA (1997) Geographic and intraspecies variation in the clinical manifestations of envenoming by snakes. In Venomous Snakes (Thorpe RS, Wuster W, Malhotra A, eds). pp. 189-204. Clarendon Press, Oxford.

17 Tsai IH, Wang YM, Chen YH, Tsai TS \& Tu MC (2004) Venom phospholipases $\mathrm{A}_{2}$ of bamboo viper (Trimeresurus stejnegeri): molecular characterization, geographic variations and evidence of multiple ancestries. Biochem J 377, 215-223.

18 Tsai IH, Wang YM \& Chen YH (2003) Variations of phospholipases $\mathrm{A}_{2}$ in the geographic venom samples of pit vipers. $J$ Toxicol -Toxin Rev 22, 651-662.

19 Scott DL (1997) Phospholipase $\mathrm{A}_{2}$ : structure and catalytic properties. In Kini, RM, ed. Venom Phospholipase $A_{2}$ Enzymes: Structure, Function and Mechanism. Wiley, Chichester, pp. 97-128. 
20 Qi YH, Gong H, Wieland SJ, Fletcher JE, Conner GE \& Jiang MS (1989) Effect of a phospholipase $A_{2}$ with cardiotoxin-like properties, from Bungarus fasciatus snake venom, on calcium-modulated potassium currents. Toxicon 27, 1339-1349.

21 ShiauLin SY, Huang MC \& Lee CY (1975) A study of cardiotoxic principles from the venom of Bungarus fasciatus (Schneider). Toxicon 13, 189-192.

22 Chang LS, Chung C, Liou JC, Chang CW \& Yang CC (2003) Novel neurotoxins from Taiwan banded krait (Bungarus multicinctus) venom: purification, characterization and gene organization. Toxicon $\mathbf{4 2 ,}$ 323-330.

23 Tsai IH, Hsu HY \& Wang YM (2002) A novel phospholipase $\mathrm{A}_{2}$ from the venom gland of Bungarus candidus: cloning and sequence-comparison. Toxicon $\mathbf{4 0 ,}$ 1363-1367.

24 Utkin YN, Kukhtina VV, Maslennikov IV, Eletsky AV, Starkov VG, Weise C, Franke P, Hucho F \& Tsetlin VI (2001) First tryptophan-containing weak neurotoxin from cobra venom. Toxicon 39, 921-927.

25 Watanabe L, Nirthanan S, Rajaseger G, Polikarpov I, Kini RM \& Arni RK (2002) Crystallization and preliminary X-ray analysis of bucain, a novel toxin from the Malayan krait Bungarus candidus. Acta Crystal D Biol Crystal 58, 1879-1881.

$26 \mathrm{Xu} \mathrm{K}$ (1986) Membrane active polypeptides from venom of Bungarus fasciatus. Biomed Res 7 (Suppl.), 89-93.

27 Chang WC, Lee ML \& Lo TB (1983) Phospholipase $A_{2}$ activity of long-chain cardiotoxins in the venom of the banded krait (Bungarus fasciatus). Toxicon 21, 163-165.

28 Schmidt JJ \& Middlebrook JL (1989) Purification, sequencing and characterization of pseudexin phospholipases $\mathrm{A}_{2}$ from Pseudechis porphyriacus (Australian red-bellied black snake). Toxicon 27, 805-818.

29 Nishida M, Terashima M \& Tamiya N (1985) Amino acid sequences of phospholipases $\mathrm{A}_{2}$, from the venom of an Australian elapid snake (king brown snake, Pseudechis australis. Toxicon 23, 87-104.

30 Takasaki C, Yutani F \& Kajiyashiki T (1990) Amino acid sequences of eight phospholipases $\mathrm{A}_{2}$ from the venom of Australian king brown snake, Pseudechis australis. Toxicon 28, 329-339.

31 Takasaki C, Kimura S, Kokubun Y \& Tamiya N (1988) Isolation, properties and amino acid sequences of a phospholipase $\mathrm{A}_{2}$ and its homologue without activity from the venom of a sea snake, Laticauda colubrina, from the Solomon Islands. Biochem J 253, 869-875.

32 Wang YM, Pong HF \& Tsai IH (2005) Unusual phospholipases $\mathrm{A}_{2}$ in the venom of two primitive tree viper Trimeresurus puniceus and Trimeresurus boneenesis. FEBS $J$ 272, 3015-3025.

33 Kuipers OP, Kerver J, van Meersbergen J, Vis R, Dijkman R, Verheij HM \& de Haas GH (1990) Influence of size and polarity of residue 31 in porcine pancreatic phospholipase $\mathrm{A}_{2}$ on catalytic properties. Protein Eng 3, 599-603.

34 Chang LS, Lin SR \& Chang CC (1998) Identification of Arg-30 as the essential residue for the enzymatic activity of Taiwan cobra phospholipase A2. J Biochem (Tokyo) 124, 764-768.

35 Yu BZ, Janssen MJW, Verheij HM \& Jain MK (2000) Control of the chemical step by leucine-31 of pancreatic phospholipase $\mathrm{A}_{2}$. Biochemistry 39, 5702-5711.

36 Qin S, Pande AH, Nemec KN, He X \& Tatulian, SA (2005) Evidence for the regulatory role of the N-terminal helix of secretory phospholipase $\mathrm{A}_{2}$ from studies on native and chimeric proteins. J Biol Chem 280, 3677336783.

37 Chang WC, Lee ML \& Lo TB (1983) Phospholipase $\mathrm{A}_{2}$ activity of long-chain cardiotoxins in the venom of the banded krait (Bungarus fasciatus). Toxicon 21, 163-165.

38 Teshima K, Kitagawa Y, Samejima Y, Kawauchi S, Fujii S, Ikeda K, Hayashi K \& Omori-Satoh T (1989) Role of $\mathrm{Ca}^{2+}$ in the substrate binding and catalytic functions of snake venom phospholipases $\mathrm{A}_{2}$. J Biochem (Tokyo) 106, 518-527.

39 Magnusson AO, Rotticci-Mulder JC, Santagostino A \& Hult K (2005) Creating space for large secondary alcohols by rational redesign of Candida antarctica lipase B. Chembiochem 6, 1051-1056.

40 Fry BG, Wuster W, Kini RM, Brusic V, Khan A, Venkataraman D \& Rooney AP (2003) Molecular evolution and phylogeny of elapid snake venom three-finger toxins. J Mol Evol 57, 110-129.

41 Ginsborg BL \& Warriner J (1960) The isolated chick biventer cervicis nerve-muscle preparation. Br J Pharmacol 15, 410-411.

42 Bulbring E (1946) Observation on the isolated phrenic nerve diaphragm preparation of the rat. $\mathrm{Br} J$ Pharmac Chemother 1, 38-42.

43 Nirthanan S, Gopalakrishnakone P, Gwee MC, Khoo HE \& Kini RM (2003) Non-conventional toxins from elapid venoms. Toxicon 41, 397-407.

44 Qian YC, Fan CY, Gong Y \& Yang SL (1998) cDNA cloning and sequence analysis of six neurotoxin-like proteins from Chinese continental banded krait. Biochem Mol Biol Int 46, 821-828.

45 Slowinski JB \& Lawson R (2002) Snake phylogeny: evidence from nuclear and mitochondrial genes. $\mathrm{Mol}$ Phylogenet Evol 24, 194-202.

46 Tsai IH (1997) Phospholipases $A_{2}$ from Asian snake venom. J Toxicol -Toxin Rev 16, 79-113.

47 Slowinski JB, Knight A \& Rooney AP (1997) Inferring species trees from gene trees: a phylogenetic analysis of the Elapidae (Serpentes) based on the amino acid sequences of venom proteins. Mol Phylogenet Evol 8, 349-362.

48 Yanoshita R, Ogawa Y, Murayama N, Omori-Satoh T, Saguchi K, Higuchi S, Khow O, Chanhome L, Samej- 
ima Y \& Sitprija V (2006) Molecular cloning of the major lethal toxins from two kraits (Bungarus flaviceps and Bungarus candidus). Toxicon 47, 416-424.

49 Singh G, Gourinath S, Sarvanan K, Sharma S, Bhanumathi S, Betzel Ch, Yadav S, Srinivasan A \& Singh TP (2005) Crystal structure of a carbohydrate induced homodimer of phospholipase A2 from Bungarus caeruleus at $2.1 \AA$ resolution. J Struct Biol 149, 264 272.

50 Coborn J (1991) Atlas of Snakes of the World, p. 430, TFH Publication Co., Neptune City, CA.

51 Bradford MM (1976) A rapid and sensitive method for the quantitation of microgram quantities of protein utilizing the principle of protein-dye binding. Anal Biochem 72, 248-254.

52 Mullis KB \& Faloona FA (1987) Specific synthesis of DNA in vitro via a polymerase-catalyzed chain reaction. Methods Enzymol 155, 335-350.

53 Maniatis T, Fritsch EF \& Sambrook J (1989) Molecular Cloning: A Laboratory Manual. Cold Spring Harbor Laboratory Press, Cold Spring Harbor, NY.
54 Litchfield JT \& Wilcoxon FJ (1949) A simplified method of evaluating does-effect experiments. $J$ Pharmacol Exp Therap 96, 99-113.

55 Thompson JD, Higgins DG \& Gibson TJ (1994) improving the sensitivity of progressive multiple sequence alignment through sequence weighting, position-specific gap penalties and weight matrix choice. Nucleicl Acids Res 22, 4673-4680.

56 Felsenstein J (1992) Phylip: The Phylogeny Interfence Package, Version 3.573. University of Washington. Department of Genetics, Seattle, WA.

57 Felsenstein J (1985) Confidence limits on phylogenies: an approach using the bootstrap. Evolution 39, 783-791.

58 Renetseder R, Brunie S, Dijkstra BW, Drenth J \& Sigler PB (1985) A comparison of the crystal structures of phospholipase $\mathrm{A}_{2}$ from bovine pancreas and Crotalus atrox venom. $J$ Biol Chem 260, 11627-11636.

59 Liu CS, Chen JP, Chang CM, Chen SW \& Lo TB (1991) Amino acid sequence of a fasciatoxin-homologue, fasciatoxin-II in the venom of Bungarus fasciatus (banded karit) J Chinese Biochem Soc 20, 33-39. 